\title{
GALACTIC FOUNTAINS
}

\author{
F. D. Kahn \\ Department of Astronomy, The University, \\ Manchester M13 9PL, England
}

\section{Summary}

The intercloud medium (ICM) is kept hot and supplied with gas by supernova remnants of Type II. The rate at which mass is added balances the rate at which gas flows away from the ICM into the galactic halo. A fountain is thus formed, since the gas only has enough energy to escape from the disk, but not from the Galaxy as a whole. The upward flow must be followed by the descent of the gas, in the form of a shocked sheet: the return flow seems to be observed in the form of intermediate velocity clouds.

These conclusions will be modified if cosmic ray pressure is important, or if there is a significant inflow of extragalactic gas into the halo.

\section{Heating the Inter-Cloud Medium}

The Population I component of the Galaxy lies within a disk extending some 100 parsecs above and below the galactic plane. It includes the brightest and most active stars, notably $\mathrm{O}$ and $\mathrm{B}$ stars, which emit powerful winds, and which eventually evolve into the progenitors of Type II supernovae. These stars consequently provide a massive input of kinetic and thermal energy into the interstellar medium close to the galactic plane.

Most of the interstellar mass is contained in molecular clouds, where the gas is cold $(T \sim 10 \mathrm{~K})$ and in atomic clouds, where it is $\operatorname{cool}(T \sim 100 \mathrm{~K})$. A small fraction of the mass is contained in HII regions, which are warm $\left(T \sim 10^{4} \mathrm{~K}\right)$; however the major fraction of the space within the disk is occupied by the inter-cloud medium (ICM) in which the density is low, with $n \sim 10^{-2} \mathrm{~cm}^{-3}$ and the temperature is high $\left(T \sim 10^{6}\right.$ $\mathrm{K})$. The gas here gains its thermal energy from shocks in supernova remnants. A more detailed description of the evolution of a remnant in the actual interstellar medium is given by Shull et al (1985).

The sudden injection of energy $E_{0}$ by a supernova explosion drives a blast wave into the surrounding medium, here modelled as being homogeneous, with density $\rho_{0}$. According to the Sedov solution the shock heading the blast has radius

$$
r=\left(\frac{2 E_{0}}{\rho_{0}}\right)^{1 / 5} t^{2 / 5}
$$

at time $t$ after the explosion. The adiabatic parameter immediately behind the shock is

$$
\kappa \equiv P / \rho^{5 / 3}=0.016 E_{0}^{2 / 5} \rho_{0}^{-16 / 15} t^{-6 / 5},
$$


and the mass enclosed by the shock is

$$
M_{s}=6.4 \rho_{0}^{2 / 5} E_{0}^{3 / 5} t^{6 / 5} \text {. }
$$

The passage of the shock heats a mass

$$
M(>\kappa)=0.10 E_{0} \rho_{0}^{-2 / 3} \kappa^{-1}
$$

so that its adiabatic parameter exceeds $\kappa$. The inner parts of a remnant experience the fastest moving shocks, and the gas there is heated so intensely that it can stay hot long enough to flow to the edge of the galactic disk before cooling. (The fate of hot gas left behind by a SNR is described by Kahn 1976.) To fix ideas, a parcel of gas in which $\kappa$ exceeds $\kappa_{*} \equiv 5 \times 10^{30}\left(\mathrm{~cm}^{4} \mathrm{gm}^{-2 / 3} \mathrm{~s}^{-2}\right)$ will not cool for $10^{6}$ years and will then still be hot enough to escape from the disk. With values $3 \times 10^{51} \mathrm{erg}$ and $2 \times 10^{-24} \mathrm{gm} \mathrm{cm}^{-3}$, for $E_{0}$ and $\rho_{0}$ respectively,

$$
M\left(>\kappa_{*}\right)=4 \times 10^{35} \mathrm{gm} \equiv 200 M_{\odot} .
$$

A reasonable estimate is that each supernova of Type II makes $200 M_{\odot}$ of gas sufficiently hot that it can escape from the disk. Such supernovae occur typically three times a century in a galaxy like ours. A given supernova provides a source of hot gas for the fountain that lasts about a million years, and so the active remnants, in this sense, are typically spaced 60 parsecs apart, with our assumed supernova rate. It seems reasonable to make the approximation that the injection of hot gas is smoothly distributed within the galactic disk, with the rate being largest close to the plane, and tapering off on either side on a length scale of order 100 parsecs.

\section{Starting the Flow into the Fountain}

The gas in the ICM has so large a sound speed that it will not be confined by gravity to the galactic disk. In fact little error is made by ignoring gravity in a description of the transition from subsonic flow, within the disk, to supersonic well outside. A steady plane parallel flow satisfies the three conservation conditions

$$
\begin{array}{cl}
\text { mass } & \rho u=\Phi(z) \\
\text { momentum } & P+\rho u^{2}=\Pi \\
\text { energy } & \frac{1}{2} u^{2}+\frac{5}{2} \frac{P}{\rho}=\epsilon ;
\end{array}
$$

here $P=$ pressure, $\rho=$ density, $u=$ velocity, $\Phi(z)=$ mass flux at height $z, \Pi=$ momentum flux.

The stagnation enthalpy $\epsilon$ remains equal to the injection energy $\epsilon_{*}$ if there is no radiative heat loss. The mass flux increases with height, starting from zero at the galactic plane and reaching some value $\Phi_{\max }$ at greater heights.

It follows from equations (6), (7) and (8) that

$$
\epsilon=\frac{5}{2} \frac{\Pi u}{\Phi}-2 u^{2}
$$

regarded as a function of $u$ the value of $\epsilon$ reaches its maximum 


$$
\epsilon=\epsilon_{\max }=\frac{25}{32} \frac{\Pi^{2}}{\Phi^{2}}
$$

when $u=5 \Pi / 8 \Phi$.

Solutions for $u$ exist for an uncooled flow provided $\epsilon_{\max }$ exceeds or is equal to $\epsilon$, or if

$$
\Phi \leq \frac{5 \sqrt{2}}{8} \frac{\Pi}{\sqrt{\epsilon}} .
$$

The sonic point is then reached at the outer limit of the region where hot gas is injected, and $\Phi=\Phi_{\text {max }}$; this condition also fixes $\Pi$ and thereby the pressure in the intercloud medium, see Figure 1. A better description allows for $\epsilon$ to decrease with $z$, since the gas at greater heights has had more time to lose energy by radiation. The gas can then pass through a sonic point, and flow away supersonically from the outer layers of the disk; see Figure 2.

\section{The Gravitational Field in the Halo}

The flow into the fountain leaves the disk with supersonic speed and its later motion is controlled by the gravitational field in the halo. A convenient model has half the gravitating mass of the Galaxy in each of Populations I and II (Sellwood and Sanders 1988), and so distributed that the rotation speed $v_{0}$ of the galactic disk is uniform. The gravitational field then has components

$$
F_{\varpi} \equiv \frac{\partial \mathcal{V}}{\partial \varpi}=-\frac{v_{0}^{2}}{2 \varpi}\left\{1-\frac{z}{\left(\varpi^{2}+z^{2}\right)^{1 / 2}}\right\}-\frac{v_{0}^{2} \varpi}{2\left(\varpi^{2}+z^{2}\right)}
$$

and

$$
F_{z} \equiv \frac{\partial \mathcal{V}}{\partial z}=-\frac{v_{0}^{2}}{2\left(\varpi^{2}+z^{2}\right)^{1 / 2}}-\frac{v_{0}^{2} z}{2\left(\varpi^{2}+z^{2}\right)}
$$

respectively in the $\varpi$-direction, perpendicular to, and the $z$-direction, parallel to the galactic axis of rotation. The leading terms on the right, in both expressions, represent the field due to the mass of Population I, and the other terms that due to Population II. When $z$ is small

$$
\frac{\partial \mathcal{V}}{\partial \varpi}=-\frac{v_{0}^{2}}{\varpi}+\frac{v_{0}^{2} z}{2 \varpi^{2}}+\cdots,
$$

and the first term here is balanced by the centrifugal force. The second term only becomes relatively important with greater height $z$, and will be ignored here. The results of this calculation can therefore only be used in the part of the halo where $|z|$ is rather smaller than $\varpi$. In the same approximation

$$
\frac{\partial \mathcal{V}}{\partial z} \sim-\frac{v_{0}^{2}}{2 \varpi} \equiv-g
$$

typically $10^{-8} \mathrm{~cm} \mathrm{~s}^{-2}$, with $v_{0}=250 \mathrm{~km} \mathrm{~s}^{-1}$ and $\varpi=10 \mathrm{kpc}$. Provided that the fountain does not rise too high, the gravitational acceleration in the $\varpi$-direction will everywhere be in approximate balance with the centrifugal force, and the $z$-component will be uniform above the galactic disk. The present treatment will not serve, though, to describe a galactic wind in which the gas inevitably is carried to large heights $z$. 


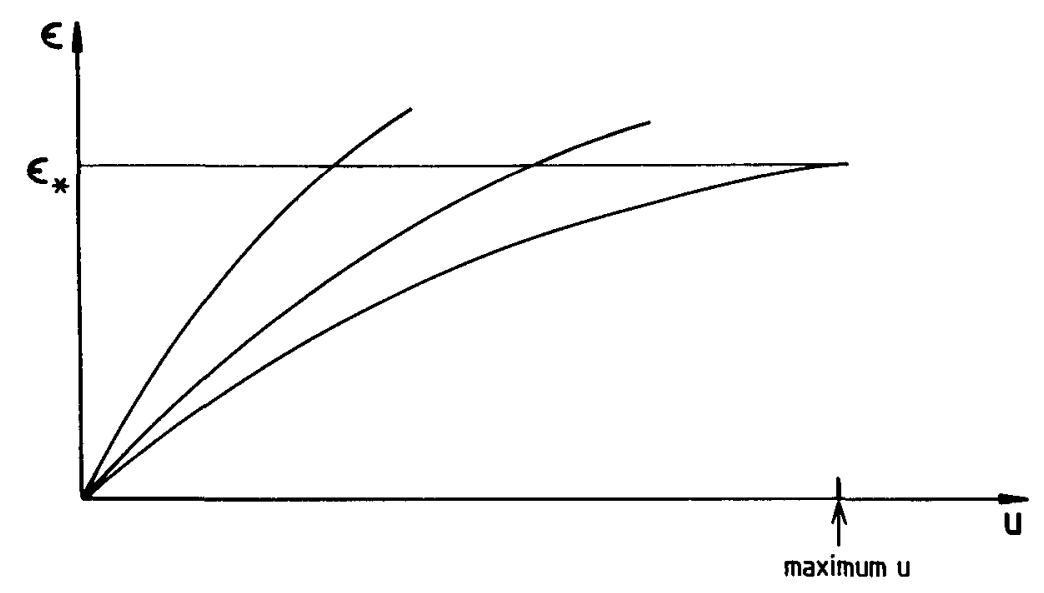

Figure 1: The successive curves show the dependence of $\epsilon$, the stagnation enthalpy, on the velocity $u$, for various values of the mass flux $\Phi$, starting on the left with small $\Phi$. The actual flow speed is determined in each case by the intersection of the curve with the horizontal line $\epsilon=\epsilon_{*}$. The last curve corresponds to the maximum value that $\Phi$ can reach. In these examples the gas is not allowed to cool radiatively. The flow is everywhere subsonic - and in the limiting case exactly sonic.

\section{The Rising Stream in the Fountain}

A simple ballistic pattern describes the upward flow in the fountain. The mass flux $\Phi$ is taken to be constant, and the upward velocity to be $u_{0}$ at the galactic plane, $z=0$. The speed of sound is negligible compared with the upward velocity, which is

$$
u=\left(u_{0}^{2}-2 g z\right)^{1 / 2}
$$

at height $z$; the density is

$$
\rho=\Phi\left(u_{0}^{2}-2 g z\right)^{-1 / 2}
$$

there. During its rising phase the fountain has an upper boundary at $z=Z(t)$, say, and

$$
\frac{d Z}{d t}=u
$$

so that the flow is still rising towards its maximum height

$$
Z_{\max }=\frac{u_{0}^{2}}{2 g}
$$

here taken to be $3 \mathrm{kpc}$, or $10^{22} \mathrm{~cm}$.

There are no effective pressure forces in the gas so that no expansion wave propagates back towards the galactic plane from the free boundary further up. An initial speed $u_{0}$ $=140 \mathrm{~km} \mathrm{~s}^{-1}$ corresponds to the $Z_{\max }$ chosen here, and the stagnation enthalpy in the flow is

$$
\epsilon_{0}=\frac{1}{2} u_{0}^{2}=10^{14} \mathrm{~cm}^{2} \mathrm{~s}^{-2}
$$


This value is also a good first estimate for the enthalpy in the hot ICM, although there will have been some radiative heat loss in the flow; the corresponding temperature is $T_{0}=3 \times 10^{5} \mathrm{~K}$. If the pressure in the ICM is $10^{-12}$ dyne $\mathrm{cm}^{-2}$, then the electron density is $n_{e, 0}=1.25 \times 10^{-2} \mathrm{~cm}^{-3}$ and the density $\rho_{0}=2.5 \times 10^{-26} \mathrm{gm} \mathrm{cm}^{-3}$. The adiabatic parameter of the ICM is

$$
\kappa=P_{0} \rho_{0}^{-5 / 3}=4.7 \times 10^{30}\left(\mathrm{~cm}^{4} \mathrm{gm}^{-2 / 3} \mathrm{~s}^{-2}\right),
$$

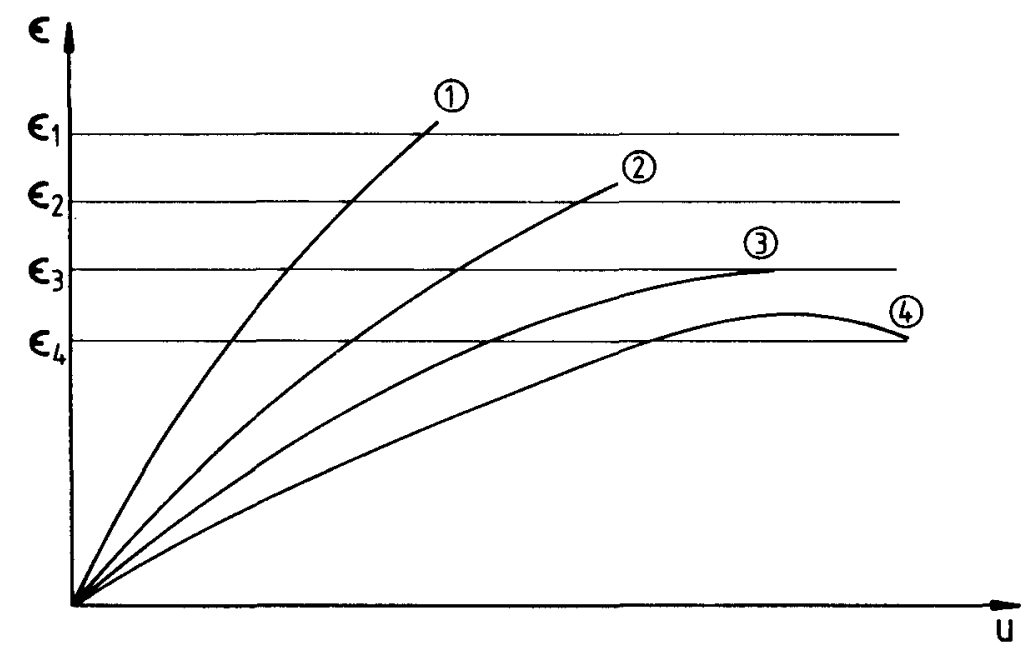

Figure 2: As in Figure 1, but with radiative cooling allowed. As a result the stagnation enthalpy decreases with height, in the order $1,2,3,4$. The contact of curve (3) with $\epsilon=\epsilon_{3}$ depicts the sonic solution, but the flow can continue to become supersonic (intersection of (4) with $\epsilon=\epsilon_{4}$ ).

in good agreement with the earlier estimate for its likely value on injection from supernova remnants. The mass flux into the halo, on each side of the disk, is

$$
\Phi=\Pi / u_{0}=P_{0} / u_{0}=7 \times 10^{-20} \mathrm{gm} \mathrm{cm}^{-2} \mathrm{~s}^{-1},
$$

and the total rate of mass loss into the fountain, from a galactic disk of radius $10 \mathrm{kpc}$, is

$$
\dot{\mathcal{M}}=4 \times 10^{26} \mathrm{gm} \mathrm{s}^{-1}=6 M_{\odot} \text { year }^{-1},
$$

again in good agreement with the earlier statement that some three supernovae of Type II are expected to occur, per century, and that each will inject some $200 M_{\odot}$ of hot gas into the ICM.

\section{Descent of the Shocked Sheet}

Once the uppermost part of the flow has reached height $Z_{\max }$ the gas will start to fall back towards the galactic plane. A well-cooled shock separates the ascending and descending parts of the fountain. The shocked gas cools rapidly because the shock speed is relatively low, and it forms a thin sheet in hydrostatic equilibrium in a rest frame moving downwards with speed $w$. 
It is convenient to use $u$, the velocity of the upward flow, as the independent variable in describing the descent of the sheet of shocked gas. With

$$
\frac{d}{d t} \equiv-w \frac{d}{d z}=\frac{w g}{u} \frac{d}{d u}
$$

the equation of conservation of mass becomes

$$
u \frac{d \Sigma}{d t}=w g \frac{d \Sigma}{d u}=\Phi(u+w),
$$

where $\Sigma$ is the surface density in the descending sheet.

The equation of motion becomes

$$
\frac{d}{d t} \Sigma w=\Sigma g-u \frac{d \Sigma}{d t}
$$

or

$$
w \frac{d}{d u} \Sigma(u+w)=\Sigma(u+w)
$$

after some algebra. From (21) and (23)

$$
\frac{d}{d \Sigma} \Sigma(u+w)=\frac{\Sigma g}{\Phi}
$$

so that

$$
\Sigma=\frac{2 \Phi}{g}(u+w)
$$

and on substituting from this relation in (21) it follows that

$$
2 w \frac{d}{d u}(u+w)=u+w
$$

or

$$
w=\frac{1}{2} u,
$$

since $u=w=0$ when $z=Z_{\max }$. The falling sheet of shocked gas has just half the speed of the upward stream, at any level. Its downward acceleration is

$$
-w \frac{d w}{d z}=-\frac{u}{4} \frac{d u}{d z}=\frac{g}{4}
$$

and the layer above the shock experiences an effective gravitational acceleration $3 g / 4$. It also follows from (21) and (25) that

$$
\frac{d \Sigma}{d t}=\frac{3}{2} \Phi
$$

and since $w=g t / 4$

$$
\Sigma=6 \Phi w / g .
$$

The pressure at the base of the shocked layer is then

$$
P_{b}=3 \Sigma g / 4=9 \Phi w / 2 .
$$

The descending gas is kept partly or fully ionized by Ly-c photons coming up from the galactic disk. This radiation originates on early type stars that are only partly shielded by their attendant HII regions. To fix ideas, a Ly-c flux of $10^{7}$ photon $\mathrm{cm}^{-2}$ $\mathrm{s}^{-1}$ will be produced, on each side of the galactic plane, by a population of naked $\mathrm{O}$ stars, each with luminosity $10^{5} L_{\odot}$, having a surface density of one star per $20000 \mathrm{pc}^{2}$. 
A descending sheet, as described earlier, reaches the vicinity of the galactic plane with a base pressure $P_{b}=2.2 \times 10^{-12}$ dyne $\mathrm{cm}^{-2}$; the flux of Ly c photons needed to keep it ionized is

$$
S_{\text {req }}=\frac{b}{m_{a}^{2}} \int_{0}^{\infty} \rho^{2} d h=\frac{b \Sigma_{0}^{2}}{2 m_{a}^{2} H}
$$

where $b=2 \times 10^{-13} \mathrm{~cm}^{3} \mathrm{~s}^{-1}$ is the effective recombination coefficient, $\Sigma_{0}=3 \Phi u_{0} / \mathrm{g}=$ $3 \times 10^{-4} \mathrm{gm} \mathrm{cm}^{-2}$ is the surface density in the sheet, $h$ measures height from the bottom of the sheet, and $H=4 c_{i}^{2} / 3 g=1.3 \times 10^{20} \mathrm{~cm}$ is the scale height within the sheet. With these parameters

$$
S_{\text {req }}=1.7 \times 10^{7} \text { photons } \mathrm{cm}^{-2} \mathrm{~s}^{-1}
$$

the shocked sheet will therefore be partly neutral if it descends on a region of the galactic disk where the surface density of unshielded $O$ stars is about equal to the standard value assumed earlier. If so the neutral hydrogen it contains will be observable by means of the $21 \mathrm{~cm}$ line, and the object would be classified as an intermediate velocity cloud. Mirabel (1989) gives an up-to-date discussion of relevant observations.

The gas returning in the fountain will never descend fast enough to be identified with the high velocity clouds, whose speeds are closer to $150 \mathrm{~km} \mathrm{~s}^{-1}$. A descending sheet will only move that fast if pushed from behind, perhaps by matter falling into the Galaxy. Doubling the downward speed raises the post-shock pressure by a factor $(4 / 3)^{2}$ $=1.78$, and the flux of Ly c photons needed for full ionization goes up to, typically, $5.4 \times 10^{7}$ photons $\mathrm{cm}^{-2} \mathrm{~s}^{-1}$.

To sum up, a shocked sheet descends to the galactic plane from the fountain with a typical speed of $70 \mathrm{~km} \mathrm{~s}^{-1}$. It is likely to be only partly ionized on returning to the galactic disk. The ionizing radiation comes from below; the neutral gas, which is cool, consequently overlies the ionized gas, which is warm $\left(T \sim 10^{4} \mathrm{~K}\right)$. This stratification is Rayleigh-Taylor unstable; if an intermediate or high-velocity cloud can be observed with the $21 \mathrm{~cm}$ line it must be partly neutral, and should therefore show an internal velocity structure, with typical speeds of $10 \mathrm{~km} \mathrm{~s}^{-1}$, resulting from the instability.

\section{Cosmic Rays in the Fountain}

Cosmic rays contribute appreciably to the energy content of the interstellar medium; some authors even argue that they provide the major part of the interstellar pressure. If this is so then the flow into the halo must be driven by cosmic rays rather than by hot gas. Substantial physical differences follow, the most important being that the cosmic ray gas cannot cool radiatively and so the energy content of the flow is less easily lost (Breitschwerdt et al 1987). But here a less extreme position will be taken.

Suppose that the flow starts into the halo with the specifications given in Section 4 , that is mass flux $\Phi$, negligible thermal energy content and upward velocity $u_{0}$, at $z=0$. Now add cosmic ray gas with pressure $\mathcal{P}_{*}$ at level $z=0$, and postulate a backgound magnetic field with uniform strength $B$ and lines of force perpendicular to the plane $z=0$. In the presence of the field the cosmic ray plasma is coupled to the thermal plasma; it can diffuse down a density gradient of cosmic rays but the diffusion speed is generally restricted to be not much larger than the Alfven speed $v_{A}$. The coupling between the thermal and relativistic plasmas is provided by Alfvén waves, which are excited whenever the speed of relative streaming exceeds the Alfvén speed and which then resonantly scatter the relativistic particles. The wave field only carries 
a relatively small fraction of the energy involved, and its contribution to the momentum flow $\Pi$ or to the rate of working $W$ will be ignored (Skilling 1970).

For the cosmic-ray assisted flow in a steady state

$$
\Pi=\Phi u+\mathcal{P}
$$

and

$$
W=\frac{1}{2} \Phi u^{2}+3 \mathcal{P}\left(u-v_{A}\right)+\mathcal{P} u,
$$

and here $\mathcal{P}$ is the cosmic ray pressure, $3 \mathcal{P}\left(u-v_{A}\right)$ gives the rate per unit area at which cosmic ray energy is convected and $\mathcal{P} u$ the rate, per unit area, at which cosmic ray pressure does work. $W$ is the total rate of working, per unit area. The gas flow slows down with height, the gas density and the cosmic ray density increase upwards, and therefore the cosmic rays diffuse downwards relative to the thermal plasma. This explains why $u$ and $v_{A}$ occur in the combination $u-v_{A}$ in relation (31).

Gravity has to be allowed for here and consequently $\Pi$ and $W$ become functions of $z$ and satisfy the equations

$$
\frac{d \Pi}{d z}=-\rho g, \quad \frac{d W}{d z}=-\Phi g,
$$

so that

$$
\frac{d W}{d \Pi}=\frac{\Phi}{\rho}=u
$$

Finally the Alfvén speed is

$$
v_{A}=\frac{B u^{1 / 2}}{\sqrt{4 \pi \Phi}} \equiv \beta u^{1 / 2} .
$$

It is now convenient to use dimensionless variables $\varpi$ and $V$, defined by

$$
v_{A}=\beta^{2} V \text { (or equivalently } u=\beta^{2} V^{2} \text { ) and } \mathcal{P}=\mathcal{P}_{*} \varpi
$$

A differential equation for $\varpi$ in terms of $V$ then follows from using relations (30), (31) and (35) in equation (33). The cosmic ray pressure is found from the solution to be

$$
\mathcal{P}=\mathcal{P}_{*} \frac{V_{0}\left(V_{0}-1\right)^{5 / 3}}{V(V-1)^{5 / 3}}
$$

and increases with decreasing $V$, or increasing $z$. The first of equations (32) can be expressed in terms of $V$ and becomes

$$
\left\{\frac{B^{2}}{2 \pi} V-\frac{\mathcal{P}_{*}(8 V-3) V_{0}\left(V_{0}-1\right)^{5 / 3}}{V^{2}(V-1)^{8 / 3}}\right\} \frac{d V}{d z}=-\frac{4 \pi \Phi^{2} g}{B^{2} V^{2}}
$$

The term in braces starts positive at the plane $z=0$, where $V=V_{0}$, and then decreases with increasing height. It will certainly reach zero while $V$ still exceeds unity and the flow is still moving upwards, but if so $d V / d z$ becomes infinite and that would be unphysical. Here then is a crucial difference which distinguishes between fountains with or without cosmic rays. The table below shows the maximum value of $\mathcal{R}_{*}$, the ratio of cosmic ray to magnetic energy density at $z=0$, which is allowable if the flow is to be able to slow down smoothly from the initial $V_{0}$ to $V$ without encountering an infinity in $d V / d z$. The last column gives the speed corresponding to $V$, in $\mathrm{km} \mathrm{s}^{-1}$. In the calculation it is assumed that $B=10^{-6}$ gauss, and that $\Phi=7 \times 10^{-20} \mathrm{gm} \mathrm{cm}^{-2} \mathrm{~s}^{-1}$ and $u_{0}=1.4 \times 10^{7} \mathrm{~cm} \mathrm{~s}^{-1}$ as before, so that 


$$
V_{0}=\sqrt{\frac{4 \pi \Phi u_{0}}{B^{2}}}=3.54
$$

The term in braces, in equation (37), is positive provided that

$$
\mathcal{R}_{*} \equiv \frac{24 \pi \mathcal{P}_{*}}{B^{2}}<\frac{36 V^{3}(V-1)^{8 / 3}}{(8 V-3) V_{0}\left(V_{0}-1\right)^{5 / 3}} .
$$

Small values of $\mathcal{R}_{*}$ correspond to a low energy density for the cosmic rays: if $\mathcal{R}_{*}$ is as small as 0.13 then the upward flow can continue almost to a complete standstill before meeting the singularity in the velocity gradient. For a more realistic value of $\mathcal{R}_{*}$, say 5.82 , the energy density due to cosmic rays would be $2.3 \times 10^{-13} \mathrm{erg} \mathrm{cm}^{-3}$, the fountain could flow without serious hindrance until its speed had halved and it had

$\begin{array}{llc}V & \operatorname{Max}^{m} \mathcal{R}_{*} & \mathrm{u} \\ \left(\mathrm{cm} \mathrm{s}^{-1}\right)\end{array}$

risen to about three quarters of height $Z_{\max }$. For still larger $\mathcal{R}_{*}$ (say 21.7) the cosmic ray energy density would be $8.6 \times 10^{-13} \mathrm{erg} \mathrm{cm}^{-3}$, rather larger than the kinetic energy density, and the flow could only rise to height $\frac{1}{2} Z_{\max }$, approximately. Clearly even a small contribution of cosmic ray energy can make a noticeable difference to the flow: a large contribution will radically change it. But cases where this contribution is large call for a special treatment of the dynamics of the transition zone from subsonic flow in the galactic disk to supersonic flow outside. Without such a calculation it is not possible to define $\mathcal{P}_{*}$ in terms of the cosmic ray energy density in the galactic disk. This problem will have to be left unsolved for the present.

\section{Discussion}

The flow into the galactic fountain is caused by the high temperature, and possibly the considerable cosmic ray pressure, in the inter-cloud medium. The gravitational field perpendicular to the galactic plane is much too small to confine the ICM within the disk. The hot gas, and the cosmic rays, therefore stream away into the halo, but there is insufficient energy available to allow the gas to escape from the Galaxy, according to this model. Consequently a return flow is set up, very probably to be identified with the intermediate velocity clouds that are observed with the $21 \mathrm{~cm}$ line. There are strong similarities between the description that has been given here and that in an earlier account (Kahn 1981). For a view with a somewhat different flavour see Bregman (1980). The treatment presented here cannot account for the observed high velocity clouds, unless there is an additional push given to the descending gas by the infall of material from outside the Galaxy. A possible configuration is shown in Figure 3. 


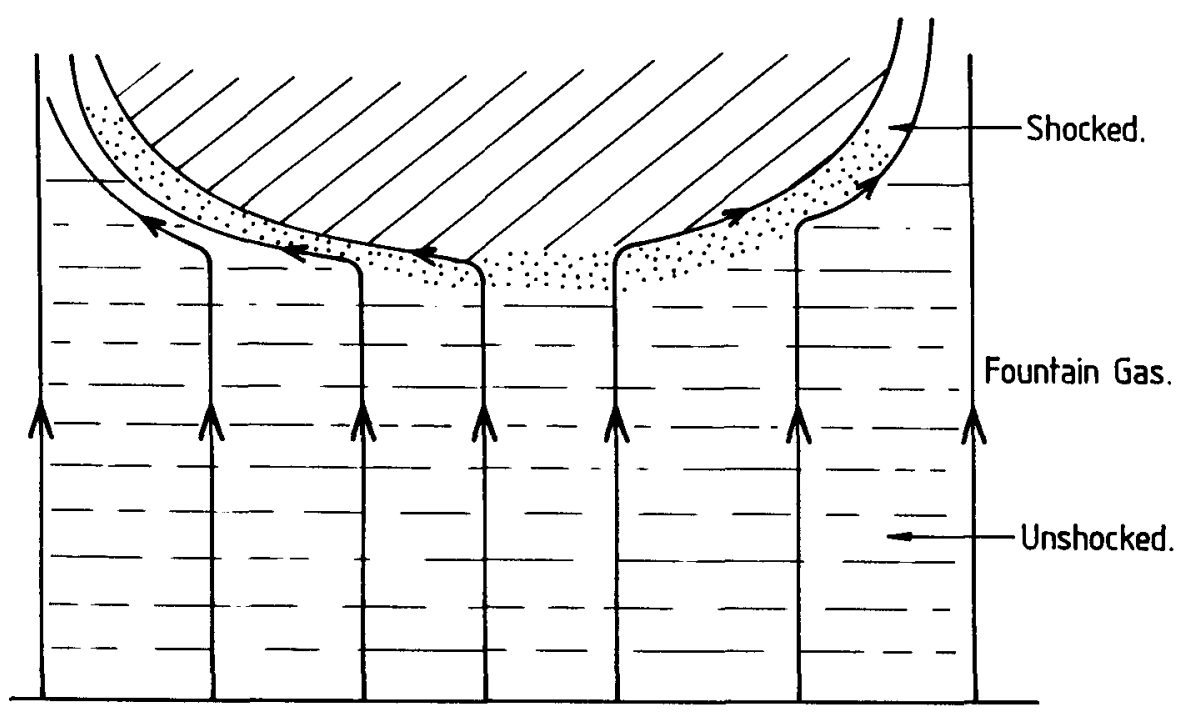

Figure 3: An infall of material (sloping hatched lines) from outside the Galaxy pushes down on the layer of shocked gas that is descending towards the galactic plane. At the same time it distorts the magnetic lines of force (shown with arrows) and compresses them. There is a possibility that such an interaction will increase cosmic ray confinement because of the creation of magnetic mirrors at the locations where the field is most compressed.

\section{References}

Bregman, J. N. 1980, Astrophys. J. 236, 577.

Breitschwerdt, D., McKenzie, J. F. and Völk, H. J. 1987, "Magnetic Field Structure of the Galaxy" in Interstellar Magnetic Fields (ed. R. Beck and R. Gräve), Springer, p. 131.

Kahn, F. D. 1976, Astron. Astrophys., 50, 145.

Kahn, F. D. 1981, "Dynamics of the Galactic Fountain" in Investigating the Universe (ed. F. D. Kahn), Reidel, p. 1.

Mirabel, J. F. 1989, "Inflow of Neutral Gas Toward the Galactic Disk" in IAU Colloquium 120 Structure and Dynamics of the Interstellar Medium (eds. G. TenorioTagle, M. Moles and J. Melnick), Springer.

Sellwood, J. A. and Sanders, R. H. 1988, Mon. Not. R. astr. Soc., 233, 611.

Shull, P., Dyson, J. E., Kahn, F. D. and West, K. A. 1985, Mon. Not. R. astr. Soc., 212, 799 .

Skilling, J. 1970, Mon. Not R. astr. Soc., 147, 1. 